\title{
Investigation of the Solid-Liquid Phase Change in the Presence of Gas Phase: Numerical Modeling and Validation
}

\author{
Ravi Govindram Kewalramani, Sebastian Pose, Ingo Riehl, Tobias Fieback \\ Institute of Heat Engineering and Thermodynamics, Chair of Technical Thermodynamics, \\ Technische Universität Bergakademie Freiberg \\ Gustav-Zeuner Str. 7, 09599 Freiberg, Germany \\ Ravi.Kewalramani@ttd.tu-freiberg.de; Sebastian.Pose@ttd.tu-freiberg.de; Ingo.Riehl@ttd.tu-freiberg.de; \\ Tobias.Fieback@ttd.tu-freiberg.de
}

\begin{abstract}
In the present work a fundamental numerical model is implemented in the open source framework, finite volume method (FVM) based computational fluid dynamics (CFD) program OpenFOAM®, to tackle the solid-liquid phase change phenomena in the presence of gas phase. The volume of fluid (VoF) method is employed to distinguish between the phase change material (PCM) and the gas phase, and the enthalpy-porosity method is adopted to capture the moving boundary phase change phenomena in the PCM. The discussed model is validated against the well documented cases in the literature i.e., 1D Stefan problem, melting of gallium and free surface thermocapillary flow with phase change. The simulation results are in very good agreement with those found in the literature. Finally experiments are performed with partially filled paraffin wax (Rubitherm ${ }^{\circledR}$ RT31) in the presence of air in an enclosure. The left and right walls of the enclosure are made of aluminium plates and maintained at different temperatures while the remaining walls are made of Plexiglas. The temporal evolution of melting front during melting obtained from the discussed numerical model is compared with experiments.
\end{abstract}

Keywords: CFD, Enthalpy-porosity method, Multiphase flows, OpenFOAM®, Solid-liquid phase change, Thermocapillary flow, VoF.

\section{Introduction}

The solid-liquid phase change in the presence of third phase (liquid or gas) is of the utmost importance in many industrial processes such as casting, welding, thermal storage systems, powder atomization, etc. Therefore the understanding of solidification and melting, its initial and thermal conditions plays a crucial role to make the process more efficient. Over the past few decades numerical simulations of solid-liquid phase change is gaining popularity in the industry not only to improve the quality of their products, but also to make cost effective manufacturing decisions [1]. A common approach for modeling such multi physical phenomena is coupling of Volume of Fluid (VoF) method with a solid-liquid phase change model in Eulerian frame.

Since the introduction of the enthalpy method by Voller et al. [2], several numerical methods, formulations and models have been found in the literature to tackle the solid-liquid phase change phenomena and especially in handling of the velocity field in the solid phase. Hu and Argyropoulos [3] reviewed various methods and stated the pros and cons of the discussed methods according to the nature of the problem and computational expenses. Rösler and Brüggemann [4] developed a numerical model to study the melting process of phase change material (PCM) in latent heat storage systems and incorporated an error function to describe the melt fraction. Miehe [5] implemented and compared the viscosity and Darcy model to investigate the twin-roll casting process of the magnesium alloy. Kasibhatla et al. [6] adopted the variable viscosity model to study the melting and settling of PCM in a capsule. More recently König-Haagen et al. [7] performed a comprehensive benchmarking of fixedgrid methods and presented a qualitative and quantitative analysis of most common energy formulations for the modeling of melting.

The VoF approach proposed by Hirt and Nichols [8] is the well-known interface-capturing technique, which uses an indicator function to mark different fluids. This approach has also been previously used for the solid-liquid phase change simulations in a multiphase system. Assis et al. [9] performed parametric CFD analysis of melting process of PCM (Rubitherm ${ }^{\circledR}$ RT27) in a partially filled spherical shell. The VoF method was employed to account for PCM-air system and the enthalpyporosity method was adopted for the solid liquid phase change. A temperature dependent density function $\varrho(T)$ was used for air phase, whereas a variable density based on Boussinesq approximation was implemented in the liquid PCM.

Shmueli et al. [10] investigated the influence of the large mushy zone constant $C$ in vertical cylindrical tube, partially filled with PCM (RT27) with a flat surface. A temperature dependent viscosity function $\mu(T)$ and variable density $\varrho$ were used in the 
liquid PCM. Two sets of thermophysical properties were examined, at first uniform values of specific heat capacity $\left(c_{p \mathrm{l}}=\right.$ $\left.c_{p s}\right)$ and thermal conductivity $\left(\kappa_{1}=\kappa_{\mathrm{s}}\right)$ for solid and liquid phase was assigned, later a small variation of thermal conductivity between phases was considered (i.e. $\left(\kappa_{\mathrm{l}} \neq \kappa_{\mathrm{s}}\right)$ ). Rösler [11] investigated close contact melting of a PCM with air above it in a capsule. The PCM was assumed to be an incompressible fluid, whereas the gas phase (air) was modeled as a compressible fluid. Similarly, Kasibhatla et al. [12] also investigated the close contact melting in a capsule using a multiphase approach and variable viscosity model to enforce the velocity in the solid region to zero.

Furthermore, Tan et al. [13] performed simulations in small scale geometries to investigate the effects of thermocapillary convection and the dynamics of the gas phase during the solidification of pure bismuth with an argon gas on the top using a multiphase approach. Saldi [14] studied the Marangoni driven free surface hydrodynamics in liquid steel weld pools during solid-liquid phase change with free surface deformation. Finally, Richter et al. [15] performed mould filling and solidification simulations; the gas phase was treated as an incompressible fluid with constant properties. A special treatment was employed to handle the Darcy-type source term at the interface between PCM and air.

As evident from the above literature research, the coupling of VoF Method with the enthalpy porosity method is an effective way to tackle such multiphysical phenomena numerically. Therefore in this study, a fundamental numerical model of solidliquid phase change in the presence of gas phase is presented. The PCM and gas phase are considered as two-phase immiscible incompressible fluid and are modeled using the $\mathrm{VoF}$ [8] approach based on interface capturing method. A fixed grid enthalpyporosity method $[16,17]$ is employed for the solid-liquid phase change. Thermophysical properties of both the fluids are constant and independent of temperature. The Boussinesq approximation is used for both the fluids (liquid PCM and air).

\section{Numerical Model}

\subsection{Governing Equations}

The solid-liquid phase change with a free surface is handled by solving the governing equations discussed in this section, using a single domain formulation. The conservation equations accounts for both the differences in fluid properties as well as surface tension forces acting at the interface. The mass, momentum and energy conservation equations are given by [13, 14, 16, 17]:

$$
\begin{gathered}
\nabla \cdot \boldsymbol{u}=0 \\
\frac{\partial \varrho \boldsymbol{u}}{\partial t}+\nabla \cdot(\varrho \boldsymbol{u u})=-\nabla p+\nabla \cdot(\mu \nabla \boldsymbol{u})+S_{B}+S_{\sigma}+S_{D} \boldsymbol{u} \\
\frac{\partial \varrho h}{\partial t}+\nabla \cdot(\varrho \boldsymbol{u} h)=\nabla \cdot(\kappa \nabla T)
\end{gathered}
$$

where $\boldsymbol{u}=(u, v, w)$ is the velocity vector, t the time, $p$ the pressure, $\varrho$ the density, $\mu$ the dynamic viscosity, $h$ the specific enthalpy, $T$ the temperature, $\kappa$ the thermal conductivity, $S_{B}$ the buoyancy source term, $S_{\sigma}$ the surface tension force term, $S_{D}$ the Darcy type source term.

\subsection{Volume of Fluid (VoF) method}

The VoF uses an indicator function $\alpha$ to mark different fluids:

$$
\alpha=\left\{\begin{array}{cl}
1 & \text { cell contains fluid } \mathrm{A} \\
0<\alpha<1 & \text { cell contains interface } \\
0 & \text { cell contains fluid } \mathrm{B}
\end{array}\right.
$$

in the present work fluid A is the PCM and fluid B is the gas phase. The volume fraction $\alpha$ transport equation can be written as:

$$
\frac{\partial \alpha}{\partial t}+\nabla \cdot(\alpha \boldsymbol{u})+\nabla \cdot\left(\boldsymbol{u}_{\mathrm{r}} \alpha(1-\alpha)\right)=0
$$


where $\boldsymbol{u}_{\mathrm{r}}=\boldsymbol{u}_{\mathrm{A}}-\boldsymbol{u}_{\mathrm{B}}$, is the relative velocity between two fluids. On the contrary to the classical VoF method originally proposed by Hirt and Nichols [8], the VoF method in OpenFOAM® uses an additional convective term also referred to as the artificial compression term (the third term in Eq.(5)) to minimize the numerical diffusion at the interface [18]. This term is only active within the cells containing interface due to its dependence on $\alpha(1-\alpha)$ where $\alpha \in(0,1)$. The relative velocity also known as the compression velocity $\boldsymbol{u}_{\mathrm{r}}$ is computed by:

$$
\boldsymbol{u}_{\mathrm{r}}=\min \left[C_{\alpha} \frac{|\varphi|}{\left|S_{f}\right|}, \max \left(\frac{|\varphi|}{\left|S_{f}\right|}\right)\right] \frac{\nabla \alpha}{|\nabla \alpha|}
$$

where $\varphi$ is the fluid flux, $S_{f}$ surface area and $C_{\alpha}$ compression coefficient. In the present work $C_{\alpha}=1$. The thermophysical properties of each fluid are expressed in terms of volume fraction $\alpha$ :

$$
\Psi=\alpha\left(\Psi_{\mathrm{A}}-\Psi_{\mathrm{B}}\right)+\Psi_{\mathrm{B}}
$$

where $\Psi$ is either $\varrho, \mu, \kappa, c_{p}$ or $\beta$.

The surface tension force term $S_{\sigma}$ in Eq. (2) is the continuum surface force (CSF) model proposed by Brackbill et al. [19] and is expressed as:

$$
S_{\sigma}=\sigma k \nabla \alpha
$$

where $\sigma$ is the surface tension and $k$ the surface curvature. The surface curvature is defined as:

$$
k=\nabla \cdot\left(\frac{\nabla \alpha}{|\nabla \alpha|}\right)
$$

\subsection{Solid-liquid phase change}

The specific enthalpy $h$ in Eq. (3) can be defined as the sum of sensible heat and latent heat, such that:

$$
h=\int_{T_{\mathrm{Ref}}}^{T} c_{p} d T+\alpha \gamma(T) L=c_{p}\left(T-T_{\mathrm{Ref}}\right)+\alpha \gamma(T) L,
$$

where $c_{p}$ is the specific heat, $T_{\mathrm{Ref}}$ the reference temperature, $\alpha$ restricts the term in fluid $\mathrm{A}(\mathrm{PCM}), \gamma(T)$ the melt fraction as a function of temperature and $L$ the latent heat of fusion.

By combining Eq. (3) and Eq. (10), we get the energy conservation equation in terms of temperature, as shown below:

$$
\frac{\partial\left(\varrho c_{p} T\right)}{\partial t}+\nabla \cdot\left(\varrho \boldsymbol{u} c_{p} T\right)=\nabla \cdot(\kappa \nabla T)-\alpha L\left[\frac{\partial \varrho \gamma}{\partial t}+\nabla \cdot(\varrho \boldsymbol{u} \gamma)\right]+T_{\operatorname{Ref}}\left[\frac{\partial\left(\varrho c_{p}\right)}{\partial t}+\nabla \cdot\left(\varrho \boldsymbol{u} c_{p}\right)\right]
$$

The melt fraction $\gamma(T) \in[0,1]$ is defined as a linear function of temperature:

$$
\gamma(T)=\left\{\begin{array}{ccl}
0 & T<T_{\text {sol }} & \text { solid, } \\
\frac{T-T_{\text {sol }}}{T_{\text {liq }}-T_{\text {sol }}} & T_{\text {sol }} \leq T \leq T_{\text {liq }} & \text { mushy zone } \\
1 & T>T_{\text {liq }} & \text { liquid, }
\end{array}\right.
$$

where $T_{\text {sol }}$ is the solidus temperature and $T_{\text {liq }}$ the liquidus temperature.

The Darcy type source term $S_{\mathrm{D}}$ in Eq. (2) is modeled using the Carman-Kozeny equation for flows in porous media [16] and is defined as: 


$$
S_{\mathrm{D}}=-\alpha C \frac{(1-\gamma)^{2}}{\gamma^{3}+\varepsilon},
$$

where $C$ is the large constant that describes the morphology of the melting front in the mushy zone and $\varepsilon=1 \times 10^{-3}$ is the small computational constant to avoid "divide by zero" error. In Eq. (13), when $\gamma=0$ the source term dominates in the cells containing solid phase and enforces the velocity in that cell to zero, and when $\gamma=1$ the source term vanishes in the cells containing liquid phase.

The thermophysical properties of each phase are expressed in terms of melt fraction ${ }^{1}$, as shown in Eq. (14), where $\psi$ is either $\varrho, c_{p}$ or $\kappa$.

$$
\Psi_{\mathrm{A}}=\gamma\left(\psi_{\mathrm{liq}}-\psi_{\mathrm{sol}}\right)+\psi_{\mathrm{sol}}
$$

The buoyancy source term in Eq. (2) is described by the Boussinesq approximation, such that:

$$
S_{\mathrm{B}}=\varrho \boldsymbol{g} \cdot\left[1-\left\{\beta \cdot\left(T-T_{\text {liq }}\right)\right\}\right]
$$

where $\boldsymbol{g}=\left(g_{x}, g_{y}, g_{z}\right)$ is the gravity vector.

The discussed model is implemented into the open source FVM based CFD software OpenFOAM®. For the pressurevelocity coupling, the PIMPLE (PISO and SIMPLE) algorithm is employed. The VoF method is solved explicitly using a modified FCT (Flux Corrected Transport) method called MULES (Multidimensional Universal Limiter with Explicit Solution) and is based on the work of Zalesak [20] with an iterative evolution of the weighting factors [21]. A first order implicit Euler scheme is employed for the temporal discretization. For the gradient terms a second order Gauss linear scheme is applied. A variety of HRS (High Resolution Schemes) discretization schemes are used to solve the divergence terms of the equations.

\section{Validation}

\subsection{Stefan problem}

The one-dimensional one-phase Stefan problem is a well-known transient heat transfer problem for directional solidification [1]. Fig. 1 shows the schematic diagram of the problem. The fluid with a melting point of $T_{\mathrm{m}}=882.15 \mathrm{~K}$ is used. The left and right boundaries are kept at constant temperatures $T_{\mathrm{c}}=822.15 \mathrm{~K}\left(<T_{\mathrm{m}}\right)$ and $T_{\mathrm{h}}=882.7 \mathrm{~K}\left(>T_{\mathrm{m}}\right)$ respectively. The initial temperature of the system is $T_{\text {init }}=882.7 \mathrm{~K}$ and the thermophysical properties of the fluid are tabulated in Table 1 . The analytical solution for the determination of interface location $x_{\mathrm{m}}(t)$ at time $\mathrm{t}$ is given by:

$$
\begin{aligned}
& x_{\mathrm{m}}(t)=2 \eta \sqrt{\alpha_{D} t}, \\
& \begin{array}{llll} 
& T_{\mathrm{m}} & \partial T / \partial y=0 & \\
\stackrel{1}{\rightarrow} T_{c} & & T_{\text {init }} & \text { Liquid } \\
\hline x_{\mathrm{m}} & \partial T / \partial y=0 &
\end{array}
\end{aligned}
$$

Fig. 1: Stefan problem.

where $\alpha_{\mathrm{D}}$ is the thermal diffusivity, and $\eta$ is the non-linear eigenvalue and is determined by solving the below transcendental equation using iterative methods.

$$
\eta \operatorname{erf}(\eta) \exp \left(\eta^{2}\right)=\frac{c_{p}\left(T_{\mathrm{m}}-T_{\mathrm{c}}\right)}{\sqrt{\pi} L}
$$

\footnotetext{
${ }^{1}$ \#Note: $\psi_{\text {liq }}=\psi_{\text {sol }}$ if not explicitly mentioned.
} 
Table 1: Thermophysical properties of the fluid.

\begin{tabular}{ccccc}
\hline $\begin{array}{c}\varrho \\
{\left[\mathrm{kg} \mathrm{m}^{-3}\right]}\end{array}$ & $\begin{array}{c}c_{p} \\
{\left[\mathrm{~J} \mathrm{~kg}^{-1} \mathrm{~K}^{-1}\right]}\end{array}$ & $\begin{array}{c}\kappa \\
{\left[\mathrm{W} \mathrm{m}^{-1} \mathrm{~K}^{-1}\right]}\end{array}$ & $\begin{array}{c}\mu \\
{\left[\mathrm{kg} \mathrm{m}^{-1} \mathrm{~s}^{-1}\right]}\end{array}$ & $\begin{array}{c}L \\
{\left[\mathrm{~kJ} \mathrm{~kg}^{-1}\right]}\end{array}$ \\
\hline 1780 & 1050 & 80 & $1 \times 10^{-3}$ & 339 \\
\hline
\end{tabular}

In the simulations a computational domain of size $150 \mathrm{~mm}$ x $2.5 \mathrm{~mm}(\mathrm{~L} \gg \mathrm{B})$ is selected and discretized with equidistant equidistant cell size of $\Delta \mathrm{x}=0.5 \mathrm{~mm}$, resulting in 1500 cells. The top and bottom boundaries are considered as adiabatic; an an empty ${ }^{2}$ boundary condition is applied on the front and back boundaries of the domain. At time $t=0$, solidification commences commences from the left boundary and continues to propagate towards the right boundary. Fig. 2 shows a comparison of numerical simulation and analytical solution for the one dimensional Stefan problem. The interface locations predicted by the simulation are in excellent agreement with the analytical solution.

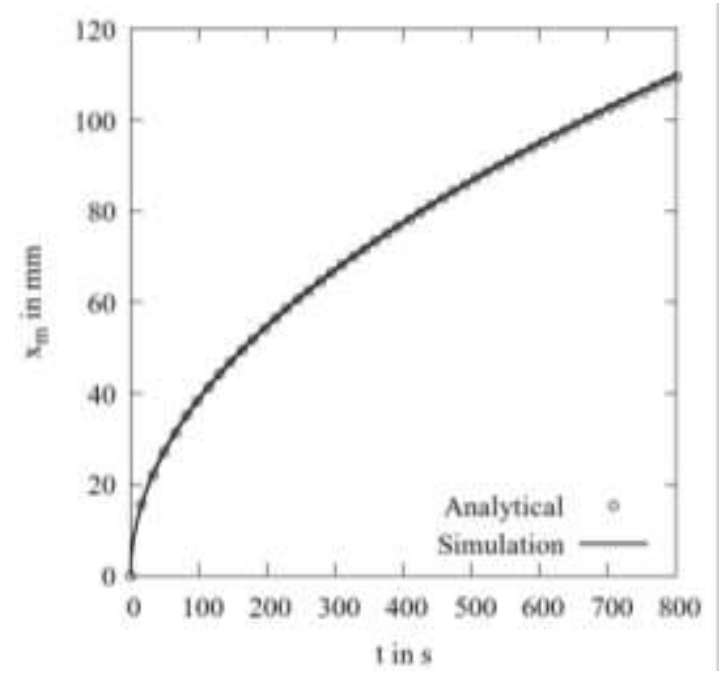

Fig. 2: Location of the interface: analytical solution and numerical simulation.

\subsection{Melting of Gallium}

The melting of gallium in a rectangular enclosure is a well-documented solid-liquid phase change experiment performed by Gau and Viskanta [22]. The computational domain is shown in Fig. 3(a) the size of the domain is $88.9 \mathrm{~mm} \times 63.5 \mathrm{~mm}$ and is divided into $42 \times 32$ hexahedral numerical cells. The left and right walls are maintained at constant temperatures $T_{\mathrm{h}}=311.15$ $\mathrm{K}, T_{\mathrm{c}}=301.45 \mathrm{~K}$ respectively. The top and bottom walls are assumed to be adiabatic. The initial temperature of the system is $T_{\text {init }}=301.45 \mathrm{~K}$. The thermophysical properties of gallium used in the simulations are given in Table 2 . Furthermore, the mushy zone constant $\mathrm{C}$ and the computational constant $\varepsilon$ in Eq. (13) are set to $1.6 \times 10^{6} \mathrm{~kg} \mathrm{~m}^{-3} \mathrm{~s}^{-1}$ and $1 \times 10^{-3}$ respectively, see Brent et al [16].

Table 2: Thermophysical properties of gallium.

\begin{tabular}{cccccc}
\hline $\begin{array}{c}Q \\
{\left[\mathrm{~kg} \mathrm{~m}^{-3}\right]}\end{array}$ & $\begin{array}{c}c_{p} \\
{\left[\mathrm{~J} \mathrm{~kg}^{-1} \mathrm{~K}^{-1}\right]}\end{array}$ & $\begin{array}{c}\kappa \\
{\left[\mathrm{W} \mathrm{m}^{-1} \mathrm{~K}^{-1}\right]}\end{array}$ & $\begin{array}{c}\mu \\
{\left[\mathrm{kg} \mathrm{m}^{-1} \mathrm{~s}^{-1}\right]}\end{array}$ & $\begin{array}{c}L \\
{\left[\mathrm{~kJ} \mathrm{~kg}^{-1}\right]}\end{array}$ & $\begin{array}{c}\beta \\
{\left[\mathrm{K}^{-1}\right]}\end{array}$ \\
\hline 6093 & 381.5 & 32 & $1.81 \times 10^{-3}$ & 80.160 & $1.2 \times 10^{-4}$ \\
\hline
\end{tabular}

The temporal evolution of the melting front obtained from simulation exhibits good agreement with experiments of Gau and Viskanta [22] as shown in Fig. 3(b). The slight deviations between the simulation and experiments can be explained as follows: (a) the melting fronts are obtained from two-dimensional simulation, whereas the experiments performed by Gau and Viskanta [22] were three-dimensional (for further information refer [5, 23]), (b) temperature independent thermophysical

${ }^{2}$ A special boundary condition in OpenFOAM® for reduced dimensions cases 
properties are used in the simulation, (c) the experiment was stopped multiple times and the melt was drained in order to track and record the melting front, which may have influenced the results.

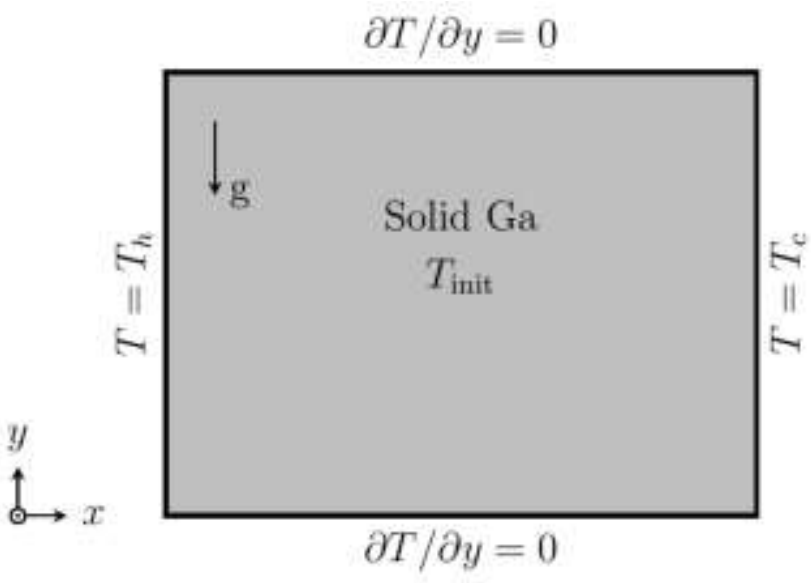

(a)

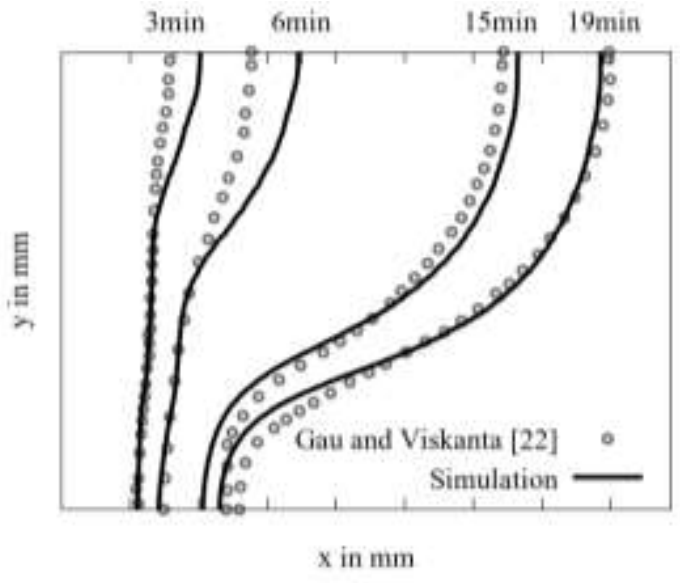

(b)

Fig. 3: Melting of gallium: (a) computational domain (b) comparison of melting fronts between simulation and experiments of Gau and Viskanta [22].

\subsection{Free surface thermocapillary flow with phase change}

A free surface thermocapillary flow with phase change in a two-dimensional rectangular container under microgravity conditions investigated by Tan et al. [13] is used for validation. For the sake of validation, the source term $S_{\sigma}$ in Eq. (8) is resolved into the normal $f_{\sigma, \mathrm{n}}$ and the tangential $f_{\sigma, \mathrm{t}}$ components $[13,14,24]$ and is expressed by:

$$
\begin{aligned}
& S_{\sigma}=\left(f_{\sigma, n}+f_{\sigma, t}\right) \delta=\left(f_{\sigma, n}+f_{\sigma, t}\right)|\nabla \alpha| \\
& =\left(\sigma k \boldsymbol{n}+\frac{\partial \sigma}{\partial T}[\nabla T-\boldsymbol{n}(\boldsymbol{n} \cdot \nabla T)]\right)|\nabla \alpha|
\end{aligned}
$$

where $\boldsymbol{n}$ is the surface normal vector and $\frac{\partial \sigma}{\partial T}$ the surface tension coefficient of temperature. Fig. 4(a) shows the computational domain of size $15 \mathrm{~mm} \times 5 \mathrm{~mm}$ and is divided into $140 \times 62$ hexahedral cells [13, 14]. The bismuth melt is filled up to height $H_{\mathrm{Bi}}=4 \mathrm{~mm}$ and the rest is filled with argon gas. The left and right walls are kept at constant temperatures $T_{\mathrm{h}}=552.55 \mathrm{~K}$ and $T_{\mathrm{c}}=540.55 \mathrm{~K}$ respectively. The top and bottom walls are enforced with a linear temperature distribution from $T_{\mathrm{h}}$ to $T_{\mathrm{c}}$, such that, the isoline of melting temperature $T_{\mathrm{m}}=544.55 \mathrm{~K}$ is at a distance $x_{\mathrm{m}}$ from the left wall. The dimensionless numbers corresponding to bismuth and argon gas are listed in Table 3 [13], where $M a$ is the Marangoni number, Ra Rayleigh number, Ca Capillary number, Ste Stefan number, Bo Biot number and $\operatorname{Pr}$ Prandtl number.

Table 3: Dimensionless numbers.

\begin{tabular}{cccccc}
\hline$M a$ & $R a$ & $C a$ & Ste & Bo & Pr \\
{$[-]$} & {$[-]$} & {$[-]$} & {$[-]$} & {$[-]$} & {$[-]$} \\
\hline 244 & 0.031 & 0.0022 & 0.033 & $1.88 \times 10^{-4}$ & 0.019 \\
\hline
\end{tabular}

The comparison of solid-liquid interface of the present work, the simulations by Tan et al. [13], and the simulations by Saldi [14] is shown in Fig. 4(b). An excellent agreement is achieved in comparison with literature. 


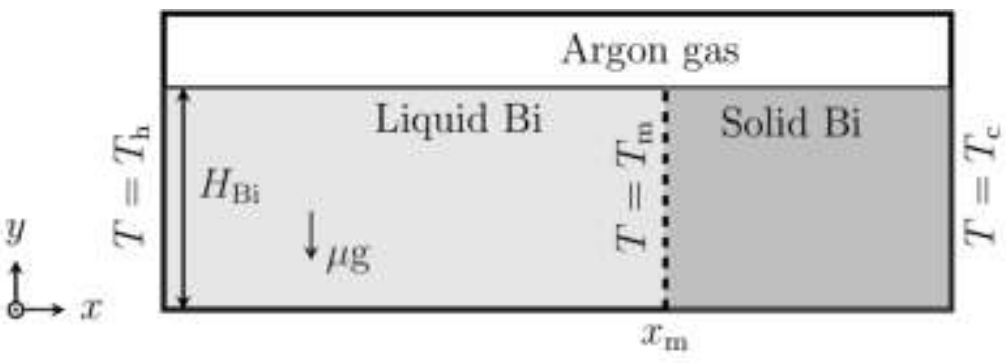

(a)

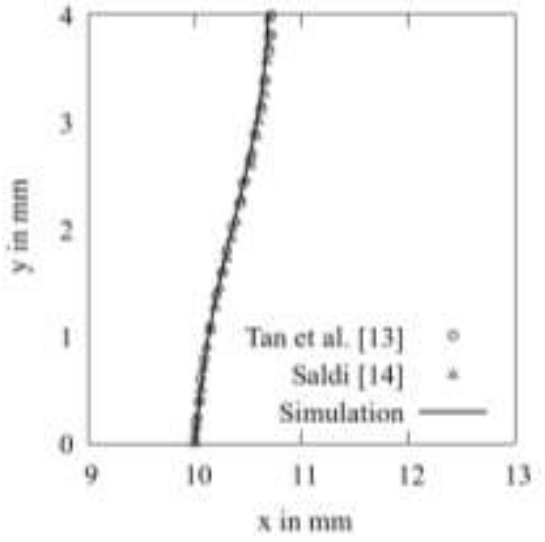

(b)

Fig. 4: Free surface thermocapillary flow with phase change: (a) computational domain (b) comparison of solid-liquid interface with literature.

\section{Experimental setup}

Experiments are conducted to further investigate the introduced model. Fig. 5 illustrates the schematics of the experimental setup adapted from $[11,22,26]$. The domain consists of a square enclosure with inside dimensions of $50 \mathrm{~mm} \times 50 \mathrm{~mm} \times 50$ $\mathrm{mm}$ made of Plexiglas. The left and right walls are made of aluminium plates which serve as heat source and heat sink respectively. A couple of multi-purpose riser pipes are screwed on the top wall of the enclosure which allows free flow of air to compensate for volume expansion/contraction during phase change. The enclosure is initially filled with paraffin wax (RT31) up to a height of $40 \mathrm{~mm}$ in liquid form through one of the riser pipes and is allowed to solidify for a certain period of time. All the walls are insulated except for the front and back walls. A DSLR camera is placed at an appropriate distance from the front wall to capture the sharp images of the temporal evolution of the melting front. A LED diffuser is placed at a suitable distance from the back wall in order to differentiate the solid and liquid phase during the melting process of the wax. Finally, all the images are processed and automated using ImageJ [25] for the evaluation of melting fronts and melt volume fraction of the paraffin wax.

\section{Results and discussions}

The thermophysical properties of RT26 to RT35 provided by the manufacturer [27] are comparable, the specific heat capacity and the thermal conductivity of the above mentioned PCMs are same and have uniform values for each (solid/liquid) phase. Shmueli et at. [10] compared their numerical results with experiments using the thermophysical properties provided by the manufacturer with a slightly varied thermal conductivities for solid and liquid phase. The thermophysical properties used in this study are adapted from the above literature and are tabulated in Table 4.

The grid convergence study is performed to investigate the influence of cell size on the melt fraction. Different grid resolutions of $2 \mathrm{D}$ computational domain have been investigated, from coarse $(200 \times 200 \times 1)$ cells to fine $(400 \times 400 \times 1)$ cells increased in steps of 25 cells in each $x$ and y direction. A grid resolution of $(275 \times 275 \times 1)$ is selected in this study. The relative errors of the melt fraction between the selected grid to the finest grid resolution are less than $2 \%$. An adaptive time stepping with Courant Number $(\mathrm{Co})$ of 0.75 is adopted. 


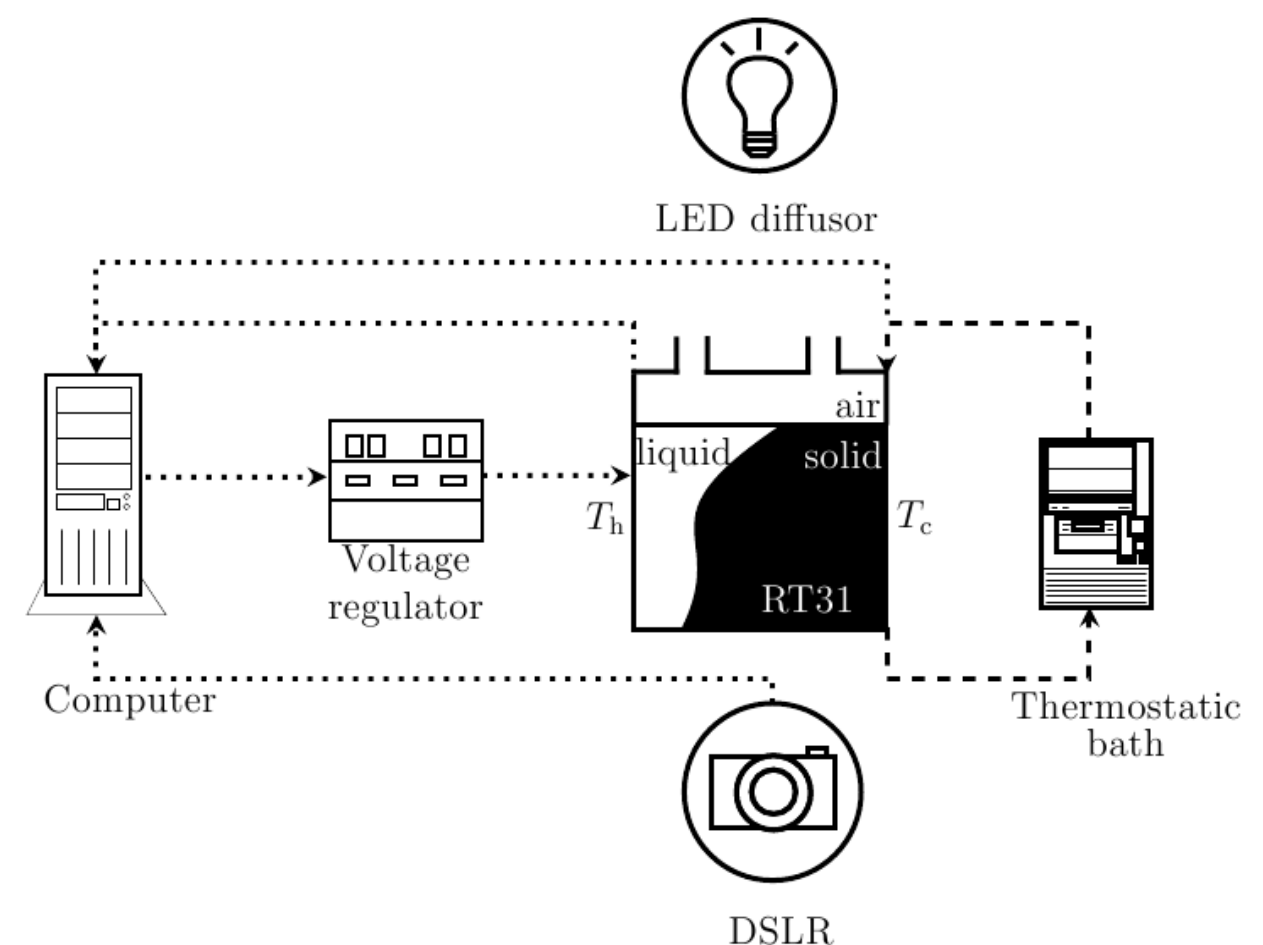

Fig. 5: Schematic representation of the experimental setup.

Table 4: Thermophysical properties used in CFD simulations.

\begin{tabular}{|c|c|c|c|c|c|c|c|c|c|c|c|c|}
\hline & \multicolumn{2}{|c|}{$\begin{array}{c}\varrho \\
{\left[\mathrm{kg} \mathrm{m}^{-3}\right]}\end{array}$} & \multicolumn{2}{|c|}{$\begin{array}{c}c_{p} \\
{\left[\mathrm{~J} \mathrm{~kg}^{-1} \mathrm{~K}^{-1}\right]}\end{array}$} & \multicolumn{2}{|c|}{$\begin{array}{c}\kappa \\
{\left[\mathrm{W} \mathrm{m} \mathrm{m}^{-1} \mathrm{~K}^{-1}\right]}\end{array}$} & \multicolumn{2}{|c|}{$\begin{array}{c}\mu \\
{\left[\mathrm{kg} \mathrm{m}^{-1} \mathrm{~s}^{-1}\right]}\end{array}$} & \multirow[t]{2}{*}{$\begin{array}{c}L \\
{\left[\mathrm{~kJ} \mathrm{~kg}^{-1}\right]}\end{array}$} & \multirow[t]{2}{*}{$\begin{array}{c}\beta \\
{\left[\mathrm{K}^{-1}\right]}\end{array}$} & \multicolumn{2}{|c|}{$\begin{array}{c}T \\
{[\mathrm{~K}]}\end{array}$} \\
\hline & sol. & liq. & sol. & liq. & sol. & liq. & sol. & liq. & & & sol. & liq. \\
\hline RT31 & 880 & 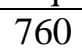 & 2000 & 2000 & 0.24 & 0.15 & - & $3.08 \times 10^{-3}$ & 160 & $7.6 \times 10^{-4}$ & 303.65 & 304.65 \\
\hline air & \multicolumn{2}{|c|}{1.1885} & \multicolumn{2}{|c|}{1006} & \multicolumn{2}{|c|}{0.0258} & & $81 \times 10^{-5}$ & - & $3.38 \times 10^{-3}$ & & - \\
\hline
\end{tabular}

Fig. 6 shows the experimental and numerical, temporal evolution of the melting front at 15, 30, 45 and 60 min respectively, for the temperature at hot and cold walls $T_{\mathrm{h}}=313.15 \mathrm{~K}$ and $T_{\mathrm{c}}=295.15 \mathrm{~K}$ respectively. The liquid paraffin close to the hot wall rises upwards and flow horizontally towards the cold melting front, where it releases heat at the upper region of the solid paraffin and descends along the cold melting front, thereby advancing the melting front rapidly in the upper region than in the lower region. The streamlines in the liquid paraffin shows that there is no movement approximately at the centre of the melt, this is because of the upwards and downwards flow of liquid paraffin close to hot wall and cold melting front (indicated by solid arrows in the bottom row of Fig. 6). It is observed that the air flow above liquid paraffin also progresses along with the melting front in a circular motion ${ }^{3}$. A secondary air flow is also observed in the region above the solid paraffin. However the strength of this secondary air flow is minuscule. In the experiment it is notable that the liquid paraffin-air interface is thicker than in simulations. This is because of the contact angle between liquid paraffin-air interface and the Plexiglas wall, which leads to the formation of meniscus near the Plexiglas wall. Furthermore, the melting fronts in the simulation appear to be sharper than in the experiment near the paraffin-air interface. This is because in experiments the melting fronts are obtained by the evaluation of photographs taken through front Plexiglas wall. Nevertheless, it is noteworthy to mention that the surface tension effects are neglected during simulations.

\footnotetext{
${ }^{3}$ \#Note: The source term used for the validation of thermocapillary flow in Sec. 3.3 is not implemented for paraffin-air simulations.
} 


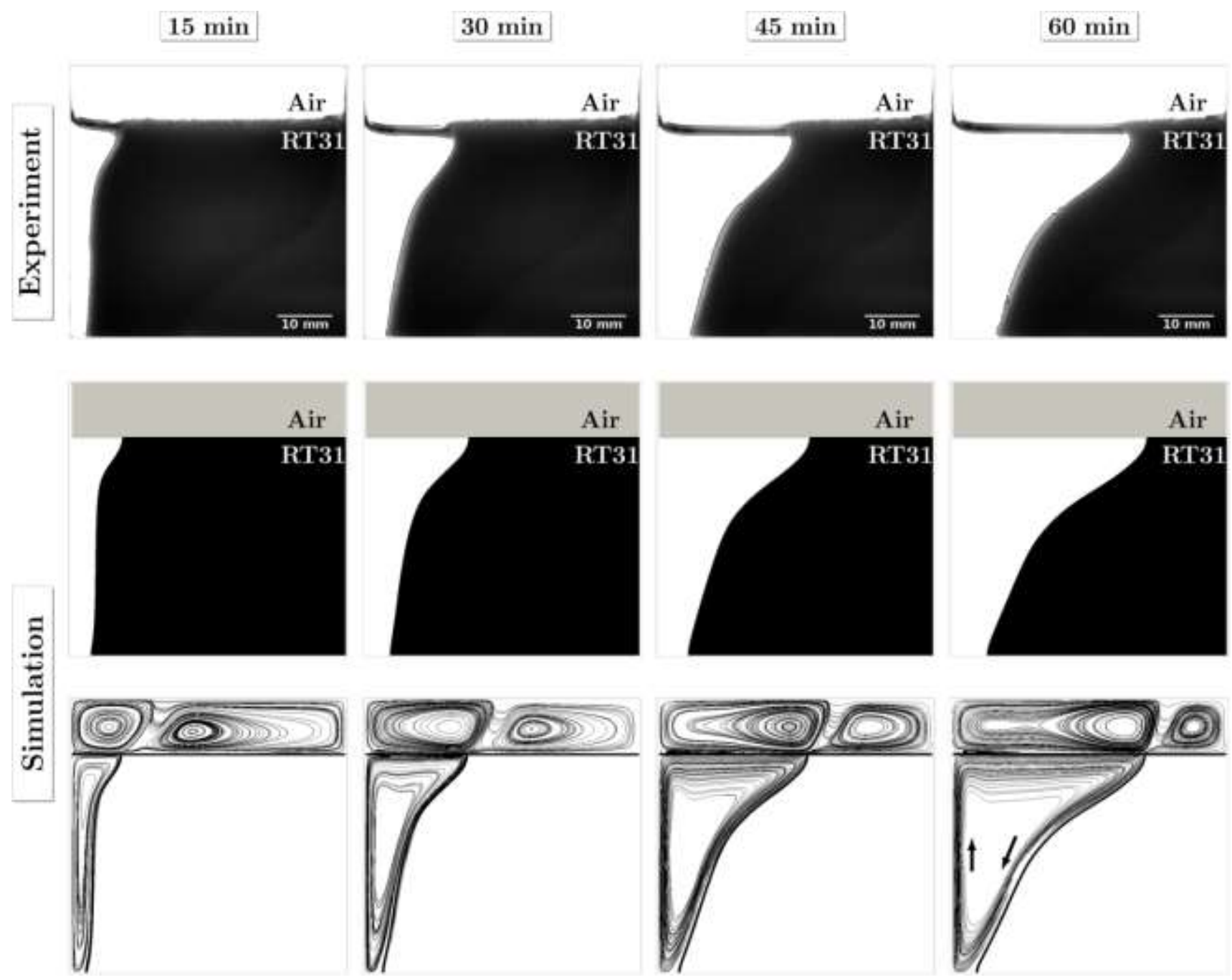

Fig. 6: Temporal evolution of melting front: experiment (top), simulation (middle) and streamlines (bottom).

Fig. 7 shows the comparison of melting fronts between experiments and simulation. The height of the liquid paraffin in the experimental domain varies due to the volume expansion throughout the phase change. Therefore, for simplicity the temporal evolution of melting fronts have been normalized to the width of the experimental domain in $\mathrm{x}$ direction and to the height of the liquid paraffin in y direction. The melting fronts obtained by the aforementioned numerical model are in good agreement with experiments. The melting fronts at the lower region advances faster in experiments than in simulations, this is because the thermal conductivity of the side Plexiglas walls also has an effect on the melting of the paraffin, whereas the influence of the side Plexiglas walls is not considered during simulations.

\section{Conclusion}

In this study, a fundamental numerical model to tackle the solid-liquid phase change phenomena in the presence of gas phase has been investigated by means of well documented case studies in the literature and experimental validation. The model is based on the use of VoF approach to account for PCM and gas phase and enthalpy-porosity method to account for solidliquid phase change in the PCM. The model has been validated using multiple references cases. The simulated results are in good agreement with the results described in the literature. 


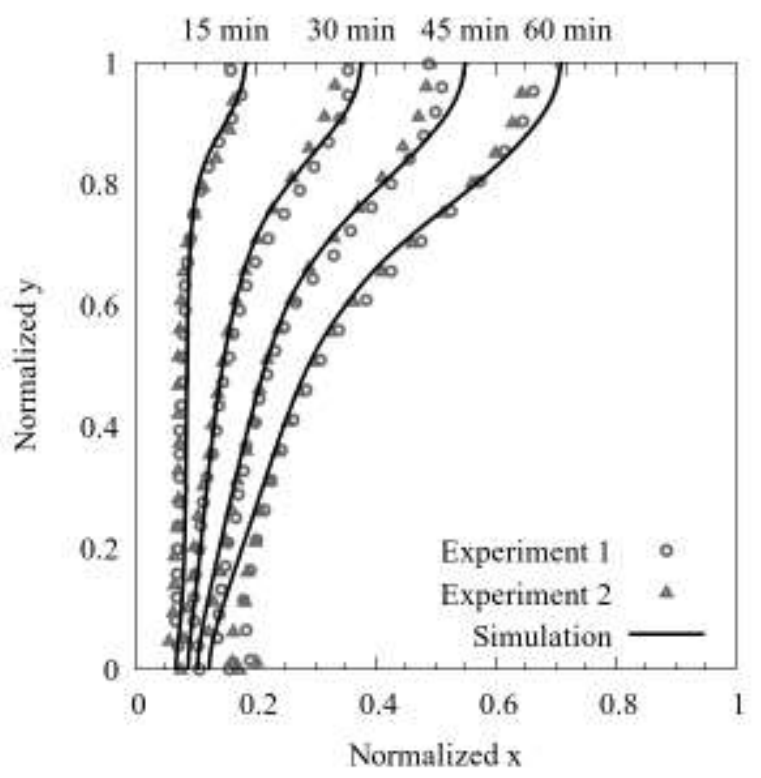

Fig. 7: Comparison of melting fronts: experiments and simulation.

To further investigate the model, experiments with paraffin wax (RT31) are conducted. Experiments are performed in a square enclosure partially filled with paraffin wax in the presence of air. The left and right walls of the enclosure are maintained at temperatures $T_{\mathrm{h}}=313.15 \mathrm{~K}$ and $T_{\mathrm{c}}=295.15 \mathrm{~K}$ respectively, whereas the remaining walls are assumed to be adiabatic. The temporal evolution of melting front obtained from simulation show a good agreement with the experiments. The small discrepancies in the results can be accounted for as follows:

- In experiments it is observed that the side Plexiglas walls also influence the melting phenomena within the enclosure, whereas in simulations these walls were not considered and are modeled as perfectly insulated.

- Temperature independent thermophysical properties are used in the simulations for PCM (solid/liquid) and gas phase.

- Only 2 dimensional simulations are carried out and the influence of the third dimension is not yet investigated.

- Finally, the errors in evaluation of photographs obtained from experiments due to volume expansion and surface tension effects.

\section{Acknowledgements}

The authors wish to acknowledge the Federal Ministry of Economic Affairs and Energy (BMWi), Germany for the financial support of MOTEVAS bearing project number 03ET1192B. Special thanks to the computer centre of Technische Universität Bergakademie Freiberg for the HPC-Cluster support.

\section{References}

[1] D. M. Stefanescu, Science and Engineering of Casting Solidification. Second edition, Springer Science+Business Media, LLC, 2009.

[2] V. R. Voller, M. Cross and N. C. Markatos, "An enthalpy method for convection/diffusion phase change," International Journal for Numerical Methods in Engineering, vol. 25, no. 1, pp. 271-284, 1987.

[3] Henry Hu and Stavros A Argyropoulos, "Mathematical modelling of solidification and melting: a review," Modelling and Simulation in Materials Science and Engineering, vol. 4, pp. 371-396, 1996.

[4] F. Rösler and D. Brüggemann, "Shell-and-tube type latent heat thermal energy storage: numerical analysis and comparison with experiments," Heat and Mass Transfer, vol. 47, no. 8, pp. 1027-1033, 2011.

[5] A. Miehe, "Numerical investigation of horizontal twin-roll casting of the magnesium alloy AZ31," Ph.D. dissertation, Technische Universität Bergakademie Freiberg, Freiberg, 2014.

[6] R. R. Kasibhatla, A. König-Haagen, F. Rösler, D. Brüggemann, "Numerical modelling of melting and settling of an encapsulated PCM using variable viscosity," Heat and Mass Transfer, Springer, 2016. 
[7] König-Haagen, E. Franguet, E. Pernot, D. Brüggemann, "A comprehensive benchmark of fixed-grid methods for the modeling of melting," International Journal of Thermal Sciences, vol. 118, pp. 69-103, 2017.

[8] Hirt and B. Nichols, "Volume of Fluid (VOF) Method for the dynamics of Free Boundaries," Journal of Computational Physics, vol. 39, pp. 201-225, 1981.

[9] E. Assis, L. Katsman, G. Ziskind, R. Letan, "Numerical and experimental study of melting in a spherical shell," International Journal of Heat and Mass Transfer, vol. 50, pp. 1790-1804, 2007.

[10] H. Shmueli, G. Ziskind, R. Letan, "Melting in a vertical cylindrical tube: Numerical investigation and comparison with experiments," International Journal of Heat and Mass Transfer, vol. 53, pp. 4082-4091, 2010.

[11] F. Rösler, "Modellierung und Simulation der Phasenwechselvorgänge in makroverkapselten latenten thermischen Speichern," Ph.D. dissertation, Universität Bayreuth, 2014. (German)

[12] R. R. Kasibhatla, A. König-Haagen, D. Brüggemann, "Numerical modelling of wetting phenomena during melting of PCM," Procedia Engineering, vol. 157, pp. 139-147, 2016.

[13] L. H. Tan, S. S. Leong, T. J. Barber and E. Leonardi, "Simulation of solidification with Marangoni effects using a multiphase approach," Progress in Computational Fluid Dynamics, vol. 6, no. 6, pp. 305-313, 2006.

[14] Z. S. Saldi, "Marangoni driven free surface flows in liquid weld pools," Ph.D. dissertation, Technische Universiteit Delft, Delft, 2012.

[15] O. Richter, J. Turnow, N. Kornev, E. Hassel, "Numerical simulation of casting processes: coupled mould filling and solidification using VOF and enthalpy-porosity method," Heat Mass Transfer, vol. 43, pp. 1957-1969, 2017.

[16] A. D. Brent, V. R. Voller, and K. J. Reid, "Enthalpy-porosity technique for modeling convection-diffusion phase change: Application to the melting of a pure metal," Numerical Heat Transfer, vol. 13, pp. 297-318, 1988.

[17] V. R. Voller and C. Prakash, "A fixed grid numerical modelling methodology for convection-diffusion mushy region phase change problems," International Journal of Heat and Mass Transfer, vol. 30, no. 8, pp. 1709-1719, 1987.

[18] H. Rusche, "Computational fluid dynamics of dispersed two-phase flows at high phase fractions," Ph.D. thesis, Imperial College of Science, Technology and Medicine, London, 2002.

[19] J. U. Brackbill, D. B. Kothe and C. Zemach, "A Continuum Method for Modeling Surface Tension," Journal of Computational Physics, vol. 100, pp. 335-354, 1992.

[20] S. T. Zalesak, "Fully multidimensional Flux-Corrected Transport Algorithms for Fluids," Journal of Computational Physics, vol. 31, pp. 335-362, 1979.

[21] S. M. Damian, "An extended mixture model for the simultaneous treatment of short and long scale interfaces, ” Ph.D. thesis, Universidad Nacional del Litoral, 2013.

[22] C. Gau and R. Viskanta, "Melting and Solidification of a Pure Metal on a Vertical Wall," Journal of Heat Transfer, vol. 108, pp. 174-181, 1986.

[23] K. Wittig and P. A. Nikrityuk, "Three-dimensionality of fluid flow in the benchmark experiment for a pure metal melting on a vertical wall," In: IOP conference series: Material science and engineering, vol. 27, 2012.

[24] T. Yamamoto, Y. Okano and S. Dost, "Validation of the S-CLSVOF method with the density-scaled balanced continuum surface force model in multiphase systems coupled with thermocapillary flows," International Journal for Numerical Methods in Fluids, vol. 83, pp. 223-244, 2017.

[25] ImageJ, https://imagej.nih.gov/ij/index.html

[26] Y. Wang, A. Amiri, K. Vafai, "An experimental investigation of the melting process in a rectangular enclosure," International Journal of Heat and Mass Transfer, vol. 42, pp. 3659-3672, 1999.

[27] Rubitherm Technologies GmbH, https://www.rubitherm.eu/en/index.php/productcategory/organische-pcm-rt. 